\title{
Intensidade Moral nas Decisões de Marketing Turístico: proposta de uma escala de mensuração
}

\author{
Moral Intensity in Tourism Marketing Decisions: a proposed scale of measurement
}

\section{La Intensidad Moral em las Decisiones de Marketing Turístico: propuesta de uma escala de medición}

\author{
Taís Alexandre Antunes Paes ${ }^{1}$
}

Francisco José da Costa ${ }^{2}$

\begin{abstract}
Resumo
A atividade turística vêm ganhando ênfase nas ultimas décadas por ser uma atividade que tem contribuído para o desenvolvimento social e econômico de várias regiões. Tal atividade apresenta importância, na medida em que, a tomada de decisão de um gestor pode gerar benefícios ou malefícios sobre a sociedade no qual o turismo está inserido. Desta forma, este trabalho apresenta como objetivo desenvolver uma escala de mensuração do construto intensidade moral considerando o contexto da tomada de decisão ética dos gestores da atividade turística. A revisão da literatura indicou que o construto intensidade moral não possuía uma escala validada para este contexto. O construto intensidade moral foi proposto por Jones (1991) que tomou como base a análise do tópico moral em si, considerando que cada indivíduo reagirá de maneira diferente em varias intensidades diante de situações éticas. Este considera que o construto intensidade moral é constituído de seis características: magnitude das consequências, consenso social, probabilidade de efeito, imediatismo temporal, proximidade e concentração do efeito. Tendo em vista as considerações apresentadas foram desenvolvidos três cenários com diferentes contextos (social, econômico e ambiental) associados a dilemas éticos da atividade turística, mais seis itens referentes as seis características da intensidade moral. Para o desenvolvimento e validação da escala foram utilizadas três amostras, duas com estudantes e uma com gestores. Os testes empíricos indicaram a consistência dos itens e dos cenários desenvolvidos, com variações de percepções segundo os tipos de cenários. Ao final são propostas idéias de aplicações da escalas e pesquisas futuras.
\end{abstract}

Palavras-Chave: intensidade moral; turismo; mensuração; validade de escala.

\section{Abstract}

\footnotetext{
${ }^{1}$ Mestre em Gestão Organizacional pela Universidade Federal da Paraíba (UFPB); Graduada em Turismo pela UFPB; Professora assistente do curso de turismo da Universidade Federal de Sergipe (UFS). E-mail: taletunes@yahoo.com.br.

${ }^{2}$ Doutor pela Fundação Getúlio Vargas (SP); Mestre em Administração pela Universidade Estadual do Ceará (UECE); Graduado em Administração de Empresas pela UECE; Professor Adjunto da Universidade Federal da Paraíba no Curso de Administração e Pós-Graduação da UFPB. E-mail: franzecosta @ gmail.com.
} 
Tourism activities are gaining emphasis in recent decades to be an activity that has contributed to the social and economic development in many regions. Such activity has importance, as the decision making of a manager can generate benefits or harms on the society in which tourism is inserted. Thus, this paper presents as objective the development of a scale for measuring the construct moral intensity considering the context of ethical decision making of the managers in tourism. The literature review indicated that the moral intensity construct didn't have a validated scale for this context. The moral intensity construct was proposed by Jones (1991) that was based on the analysis of moral topic in itself, considering that each individual will react differently at various intensities in the face of ethical situations. This author considers that the moral intensity construct is composed of six characteristics: magnitude of consequences, social consensus, probability of effect, temporal immediacy, proximity and concentration of effect. Based in this considerations this study presents the development of three scenarios based in different contexts (social, economic and environmental) associated with ethical dilemmas of tourism and six items about the six characteristics of moral intensity. For the development and validation of the scale we used three samples, with two students and one with managers. The empirical tests indicate the consistency of the items and scenarios developed, with variations in perceptions according to the types of scenarios. In addition ideas are proposed at the end of the scales of applications and future research.

Keywords: moral intensity; tourism; measurement; scale validity.

\section{Resumen}

Las actividades turísticas están ganando importancia en las últimas décadas de ser una actividad que ha contribuido al desarrollo social y económico en muchas regiones. Esta actividad tiene importancia, ya que la toma de decisiones de un gerente puede generar beneficios o los daños en la sociedad en el turismo. Por lo tanto, este trabajo presenta un objetivo de desarrollar una escala para medir la intensidad de la construcción moral teniendo en cuenta el contexto de la tomada de decisiones éticas de los directivos del turismo. La revisión de la literatura indica que la construcción de la intensidad moral no tiene una escala validada para este contexto. La construcción de la intensidad moral fue propuesta por Jones (1991) que se basó en el análisis del tema moral en sí mismo, teniendo en cuenta que cada individuo reacciona de manera diferente en distintas intensidades en la cara de situaciones éticas. Este considera la construcción de la intensidad moral se compone de seis características: magnitud de las consecuencias, el consenso social, la probabilidad de efecto, la inmediatez temporal, la proximidad y la concentración de efecto. Teniendo en cuenta las consideraciones presentadas três cenarios fueron desarrollados en diferentes contextos (sociales, económicos y ambientales) relacionados con los dilemas éticos del turismo, seis puntos sobre las seis características de intensidad moral. Para el desarrollo y validación de la escala se utilizaron três muestras, con dos estudiantes y uno con los directivos. Las pruebas empíricas indican la consistencia de los objetos y escenarios desarrollados, con variaciones en las percepciones de acuerdo a los tipos de escenarios. Las ideas se proponen al final de la escala de las aplicaciones y la investigación futura.

Palabras clave: la intensidad moral; el turismo; la medición; la validez de la escala. 


\section{Introdução}

Este artigo apresenta como tema principal o estudo da ética, em especial, a intensidade moral, perspectiva baseada no modelo de questões contingenciais de tomada de decisões éticas de Thomas Jones (1991), e toma como contexto de aplicação a atividade turística. Estudos envolvendo a intensidade moral vem sendo amplamente desenvolvidos na literatura de ética nos negócios, e esforços mais intensos tem sido realizados no sentido de identificar a forma como a avaliação de dilemas éticos por agentes do sistema de trocas, podem facilitar no entendimento da formação das intenções e das práticas aperfeiçoadas do ponto de vista moral. Neste sentido, a compreensão da moral dos gestores da atividade turística permite que melhores condutas ocorram por parte destes beneficiando a atividade turística.

Na literatura especializada, sobre ética em marketing, é possível observar a tendência no desenvolvimento de pesquisas de base quantitativa, com abordagem de amostras razoavelmente grandes e que viabilizam a operacionalização de procedimentos estatísticos de testes e relacionamento entre variáveis. Diante deste contexto ganha relevância a questão da mensuração dos construtos envolvidos nas pesquisas, uma vez que estas não abordam especificamente o construto intensidade moral. Além disso, a literatura pertinente sobre a ética, relacionada a atividade turística, apresenta poucos trabalhos vinculados a utilização de cenários e escalas do ponto de vista ético.

Desta forma, este estudo propõe o desenvolvimento de uma escala de mensuração da intensidade moral, na tentativa de gerar uma contribuição que viabilize a realização de estudos empíricos no campo específico do setor turístico, cujas pesquisas envolvendo a intensidade moral não são comuns.

A proeminência dada aos aspectos éticos dos gestores, na atividade turística, ocorre devido ao setor turístico apresentar potencial de desenvolvimento e influência social (HIGGINSDESBIOLLES, 2006; HOLJEVAC, 2008). A ética dos gestores que tomam decisões de marketing no setor turístico, apresenta importância, pois está relacionada a um setor que apresenta consequências muito maiores do que apenas contribuir para a economia de uma localidade, uma vez que se relaciona com a sociedade.

Neste sentido, as decisões e ações do gestor de turismo recebem acuidade por influenciar direta ou indiretamente a sociedade em que está inserida, tornando-o um agente social cujos aspectos éticos são evidenciados em seus comportamentos, atitudes e relacionamentos (WEEDEN, 2002). 
Considerando os elementos apresentados, este artigo esta dividido da seguinte forma: inicialmente é apresentada uma discussão sobre o construto de base da escala, que é a intensidade moral. Em seguida, são tecidas algumas considerações sobre os procedimentos convencionais e contemporâneos de mensuração. Na terceira etapa é descrito o processo operacional de desenvolvimento da escala, e, ao final, são apresentadas as considerações finais do estudo.

\section{Modelo da Intensidade Moral e a Atividade Turística}

A década de 1980 foi responsável pela difusão do conhecimento na área da ética em marketing, permitindo que houvessem diversos debates e discussões relevantes. Em meio à agitação de pensamentos e estudos que foram desenvolvidos neste período, alguns pesquisadores como Ferrell e Gresham (1985), Treviño (1986) e Hunt e Vitell (1986), desenvolveram modelos teóricos destinados a prever como as decisões éticas ocorrem (HERINGTON; WEAVEN, 2007). Estes modelos, em um contexto geral estabeleciam a relação de influência entre as filosofias morais e a tomada de decisões éticas pelos indivíduos. Por tal pressuposto, os mesmos passaram a ser considerados de grande importância para a compreensão do processo decisório envolvendo questões éticas (VITELL; SINGHAPAKDI; THOMAS, 2001). Neste sentido, os mesmos são considerados como referência nos estudos da ética em marketing.

Baseados nestes estudos, pesquisas como as de Jones (1991), Vitell, Singhapakdi e Thomas (2001), Cherry e Fraedrich (2002) e Herington e Weaven (2007) foram desenvolvidas tendo por fundamento os intentos dos modelos de tomada de decisões éticas. Entre estes estudos empíricos, destaca-se a investigação de Thomas Jones (1991), que, ao se embasar em tais modelos teóricos, insere a perspectiva da existência de níveis de intensidade moral avaliados durante as decisões éticas dos indivíduos

Em sua publicação de 1991, Jones constatou que os modelos teóricos individuais de tomada de decisões éticas nas organizações enfatizavam pouco as características das questões éticas em si. A percepção de Jones (1991) foi a de que as questões morais variam em termos de sua intensidade moral e de que a proposição de um modelo com as características do tópico moral podem fornecer uma maior compreensão sobre o processo ético.

Tendo em vista as percepções citadas, o autor delineia as seis características das questões morais (magnitude das consequências, consenso social, probabilidade de efeito, imediatismo temporal, proximidade e concentração do efeito), que se relacionam positivamente com o 
comportamento e a tomada de decisão moral. A perspectiva do autor é de que cada indivíduo reage de forma diferenciada com relação a situações que incluem elementos morais. Tais características do tópico moral, em conjunto, formam a chamada intensidade moral que estão apresentadas a seguir:

- $\quad$ O componente magnitude das consequências é definido como sendo o somatório de malefícios ou benefícios gerados sobre as vítimas ou beneficiários do ato moral em questão. $\mathrm{O}$ autor justifica este ponto exemplificando que um ato que causa a morte de seres humanos apresenta uma maior magnitude de consequências do que uma ação que gere sobre uma pessoa um efeito como um ferimento, por exemplo;

- $\quad$ O componente consenso social pode ser entendido como a conformidade social sobre a proposição de um ato ser considerado bom ou ruim. Jones (1991) inclusive argumenta que é difícil uma pessoa agir eticamente se ela não sabe o que a ética prescreve em uma situação, mas o consenso social é uma dimensão que contribui para a redução da ambiguidade existente;

- $\quad$ Outro atributo ou componente da intensidade moral é a probabilidade de efeito da ação moral, que se refere ao grau de probabilidade de uma determinada ação de conteúdo moral relevante ao sujeito venha realmente ocorrer. Para o autor, maior probabilidade de gerar prejuízo, por exemplo, promove um maior cuidado por parte do decisor antes de agir;

- $\quad$ O quarto componente da intensidade moral é o imediatismo temporal, que se refere a quando o efeito da ação poderá ter seus efeitos realizados. Segundo Jones (1991), esta dimensão faz parte da intensidade moral porque as pessoas tendem a minimizar a percepção de intensidade do impacto dos eventos que ocorrerão no futuro, de modo que quanto maior a distância no tempo entre decisão e efeito, maior a disposição do agente de tomar a decisão potencialmente menos ética;

- $\quad \mathrm{O}$ quinto componente da intensidade moral é a proximidade, que se refere à proximidade (social, cultural, psicológica ou física) que o agente moral tem dos seus beneficiário ou vítimas da ação em questão. Jones (1991) entende que quanto mais afastadas (socialmente, culturalmente, psicologicamente ou fisicamente) forem às pessoas, menores serão as preocupações com estas no momento do desempenho das decisões éticas.

- $\quad$ O sexto e último componente é a concentração do efeito, que se refere à amplitude do impacto sobre as pessoas, e é uma função inversa ao número de indivíduos afetados pela ação de 
uma dada magnitude. Ou seja, para o autor, se o efeito de uma decisão é concentrado uma pessoa, a avaliação é diferentes do que a situação em que o efeito recairia sobre muitas pessoas.

Segundo Jones, estes seis elementos apresentam relacionamentos com a intensidade moral de modo que o aumento de um dos componentes da intensidade moral contribuiria para o também aumento do construto intensidade moral. Ou seja, bastaria a alteração positiva ou negativa de um ou mais componentes para que o seu, ou os seus efeitos recaiam também sobre o construto em sentido mais amplo.

O modelo integrativo de Thomas Jones envolve, além do construto da intensidade moral, os construtos ambiente, reconhecimento das questões morais, realização do julgamento moral, estabelecimento da intenção moral, realização do comportamento moral, significado do outro, moderadores individuais, moderadores situacionais e oportunidade. Além disto, existem ainda 4 tipos de moderadores, que são o significado do outro, os moderadores individuais, os situacionais, e a oportunidade associada.

Os contextos de determinação mais genéricos que incidem sobre o reconhecimento do tópico moral são os ambientes social, cultural, econômico e organizacional. Esta incidência denota que, em geral, as questões morais são provenientes destes ambientes. O modelo pode ser visualizado na figura 1.

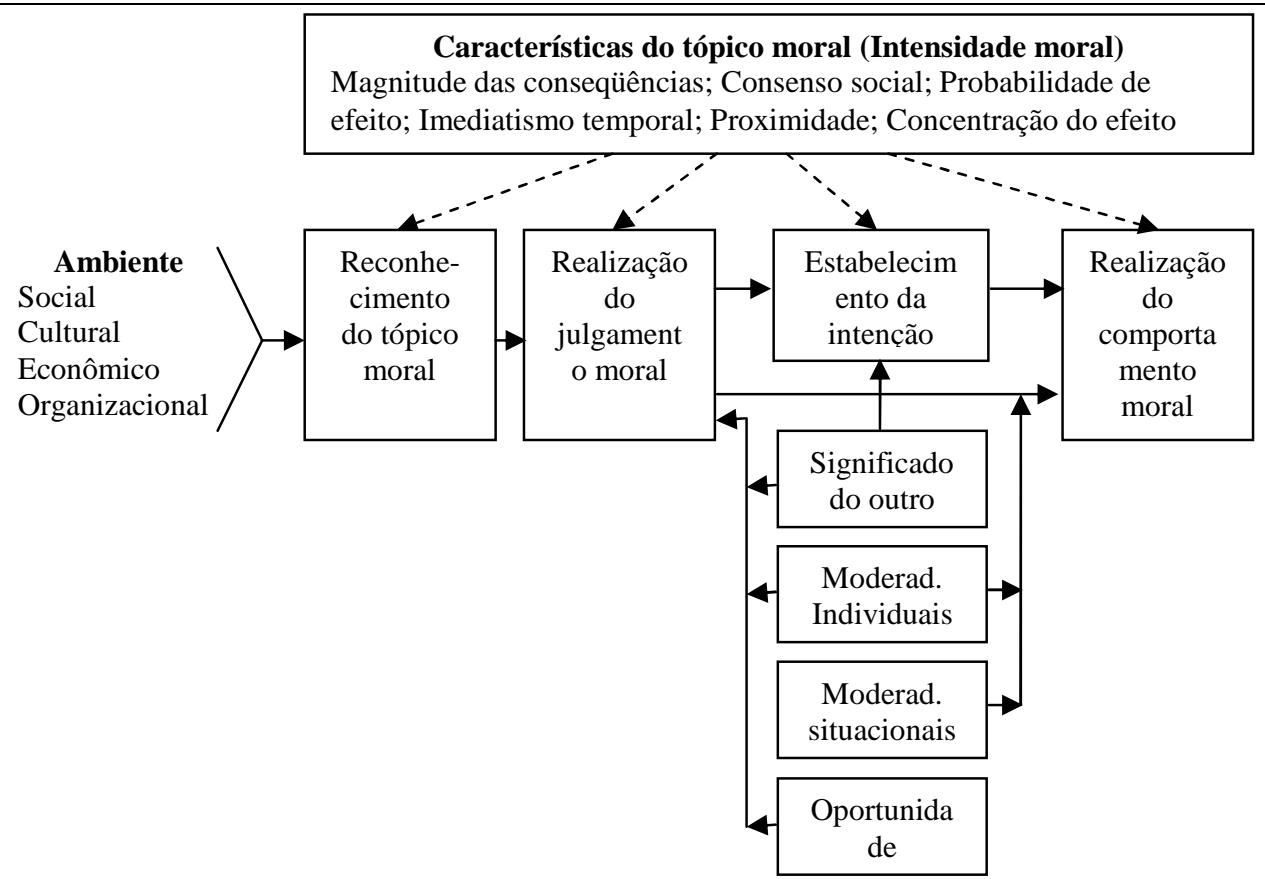

Figura 1 - Modelo da questão contingencial de tomada de decisão Fonte: Jones (1991) 
Ao se analisar as seis características, das questões morais, propostas por Jones (1991) é possível verificar que tais componentes morais estão presentes em várias formas no cotidiano das pessoas. Constantemente, avaliações éticas são necessárias diante de contextos circunstanciais da vida diária e em tais situações as decisões éticas ocorrem considerando vários aspectos. Não diferente de outros setores, a atividade turística também vem requerendo atenção com relação aos conteúdos éticos, pois lidam com a atividade humana, e envolvem a necessidade de posturas mais éticas, tanto na perspectiva do demandante quanto na do ofertante dos serviços turísticos.

Neste sentido, investigações sobre os aspectos éticos, do ponto de vista dos gestores que tomam decisões de marketing na atividade turística, podem ser entendidos como possuindo uma grande relevância, visto que os mesmos são agentes sociais que exercem atividades que contribuem (potencialmente) para a melhoria da sociedade e não apenas para a geração de benefícios econômicos.

Como atividade econômica e promotora de serviços, o turismo oferece produtos com características de intangibilidade, inseparabilidade (o produto é adquirido e consumido no mesmo instante), heterogeneidade e perecibilidade (o produto não pode ser estocado), o que o configura como um serviço (estas características diferem das dos produtos manufaturados, que permitem outras avaliações quanto à produção e consumo).

Nestes termos, diferentemente do produto físico, o produto turístico baseia-se na experiência vivenciada por seus consumidores, e os consumidores dos produtos turísticos baseiam suas compras na promessa de vivência de determinadas emoções. O processo de tomada de decisão de consumo é complexo, pois envolve decisões de onde ir e o que fazer com avaliações e percepções fundamentadas em um alto risco, uma vez que os mesmos não têm noção exata de como será a viagem até que a mesma seja vivenciada (SMALLMAN; MOORE, 2010).

Em algumas situações, a compra do produto turístico, como por exemplo, o serviço de uma companhia aérea, apresenta o elemento tangível que é o transporte, porém não se sabe se o serviço oferecido por esta empresa estará condizente com o valor pago e a expectativa de um bom serviço vendido. Por esta razão, a maioria dos produtos desta atividade é considerada de alto envolvimento e requer extensivas tomadas de decisões, devido aos elevados custos 
monetários e não-monetários a ela relacionados (SIRAKAYA; WOODSIDE, 2005).

Para efeito de fundamentação do estudo empírico, optou-se por abordar alguns dilemas éticos dos mais comuns na atividade turística. Do que se depreendeu da literatura especializada sobre esta atividade, o dilema ético mais recorrente está relacionado às transações econômicas convencionais, que perpassam os diversos setores empresariais. Por outro lado, além dos dilemas econômicos, emergiram a partir dos anos de 1990 diversos questionamentos sobre os impactos ambientais da atividade turística, associados principalmente ao uso de ambientes preservados para atividade turística e à destruição ambiental para construção de equipamentos turísticos (HOLDEN, 2003), o que promoveu uma tendência à práticas de turismo ajustadas ao conceito de turismo sustentável (LANSING; VRIES, 2007).

Além dos dilemas econômicos e ambientais, uma terceira categoria de problema ético que emergiu na atividade turística concerne aos potenciais danos sociais provocados pela atividade, com destaque para o potencial isolamento de comunidades nativas de espaços turísticos para abrigar equipamentos de propriedade privada ou o caso clássico da exploração sexual do chamado turismo sexual (OPPERMANN, 1999.).

Considerando a proposição de desenvolvimento de uma escala para mensurar a intensidade moral no contexto do turismo, e considerando as especificidades das práticas de mensuração envolvendo construtos de ética, estas três modalidades de dilema ético (econômica, social e ambiental) foram adotadas para servir de base de referência para o desenvolvimento da escala. Os detalhes estão indicados no item 4 deste artigo, porém antes serão tecidas algumas considerações sobre mensuração, expostos no item seguinte.

\section{Mensuração}

A decisão da construção da escala está respaldada na percepção de que as atuais escalas de mensuração da intensidade moral não foram desenvolvidas sobre o ponto de vista dos gestores da atividade turística. Tal consideração está fundamentada nas pesquisas bibliográficas realizadas durante este estudo. Estas pesquisas não indicaram estudos de turismo que estivessem analisando este enfoque.

Alguns autores, em especial Churchill (1979) e Rossiter (2002), por exemplo, propuseram etapas para a construção de escalas e serviram de base para pesquisas empíricas (ex. WEEB; 
MOHR; HARRIS, 2008) com construtos de natureza refletiva. A clássica proposta de Churchill (1979), ao pressupor a natureza dos itens como sendo refletiva, indica que a sua mensuração se daria a partir de múltiplos itens com decisões respaldadas na revisão da literatura, análise fatorial exploratória e análise de confiabilidade. Fundamentado nas proposições de Churchill (1979), mas com a intenção de ampliar a proposição para outros tipos de construto, Rossiter (2002) delimitou o seu modelo de construção de escalas em 6 passos principais (Definição do construto, classificação do objeto, do atributo, definição do construto, identificação do respondente, formação da escala e enumeração).

Em anos mais recentes este paradigma vem sendo fortemente contestado, em especial com propostas de revisões no modelo geral de mensuração (ROSSITER, 2002), com a adoção de itens com relação formativa em relação ao construto (DIAMANTOPOULOS; WINKLEHOFER, 2001), e também com a adoção de apenas um item como método de verificação (DROLET; MARRISON, 2001; KWON; TRAIL, 2005).

Operacionalizações do construto em outras pesquisas relacionadas a ética em marketing utilizaram comumente a estratégia de um único item como as pesquisas de Singhapakdi, Vitell e Kraft (1996), Frey (2000), Marta e Sighapakdi (2005) e McMahon e Harvey (2006). Adicionalmente, a literatura especializada sobre mensuração sinaliza a consistência desta estratégia de verificação.

Todo este conjunto de inovações foi considerado na proposição da escala deste artigo. Em adição, e considerando que a escala está relacionada à ética dos gestores na atividade turística, foi utilizado como suporte cenários, que, conforme Wason, Polonsky e Hyman (2002), são pequenas descrições que permitem aos entrevistados estarem diante de uma situação de contexto ético em que tem de tomar um posicionamento. O item seguinte descreve os procedimentos aqui adotados com maiores detalhes.

\section{Desenvolvimento da Escala}

Tendo realizado os procedimentos exploratórios anteriores, especialmente a revisão sistemática da literatura e definido o tipo de construto, é necessário agora indicar a forma como foi desenvolvida a escala para avaliar a intensidade moral dos gestores na atividade turística. 


\subsection{Elaboração dos cenários}

A utilização de cenários é frequentemente observada nas pesquisas de ética em marketing, devido a sua aplicabilidade e benefícios. Sobre este aspecto, Wason, Polonsky e Hyman (2002) evidenciam algumas reservas relativas à construção de cenários que devem ser seguidas. Estes autores ressaltam a inclusão nos cenários de variáveis relevantes de maneira que as respostas aos itens da escala reflitam de forma clara e imparcial o que pode ser entendido do enunciado. Outro ponto importante refere-se à conotação de situações reais nos cenários. Além disso, a escala deve conter um número razoável de cenários, não sendo muito longos para não cansar o leitor, e tão pouco muito curtos, de forma a não conterem os elementos necessários para uma boa interpretação dos respondentes. Cuidados adicionais devem incidir em relação aos vieses que podem ocorrer na forma como os cenários foram construídos, pois uma construção deficiente pode levar os respondentes a interpretar elementos que estão fora do propósito da escala. Além disso, os cenários devem captar variações existentes na população de interesse, sem trazer respostas já definidas.

Considerando as recomendações acima foram desenvolvidos três cenários, abordando circunstâncias éticas em três esferas de dilema ético: esfera social, esfera econômica e esfera ambiental. A criação destes cenários esteve fundamentada no entendimento de que a atividade turística faz parte de um sistema integrado de trocas, e que um conjunto de influências está representado de diferentes maneiras.

Os cenários desenvolvidos, considerando situações éticas comuns à atividade turística, encontram-se no quadro 1 , sendo o primeiro cenário referente ao contexto ético em nível social, o segundo ao contexto econômico e o terceiro ao contexto ambiental. A pretensão associada aos cenários foi de que estes sirvam de base para a aferição das seis dimensões da intensidade moral. Para uma melhor visualização de como estas características foram abordadas nos cenários, foi construído o apêndice 1 . 


\section{Cenário 1:}

Uma famosa empresa turística há vários anos apresenta como principal destino turístico passeios a uma pequena comunidade litorânea, que além de propiciar o contato com a natureza, permite momentos de tranquilidade a seus visitantes. Esta empresa é muito bem quista pelos moradores desta comunidade por proporcionar a estes a geração de renda tão necessária para o seu desenvolvimento. Este sentimento amigável é que tem incentivado esta empresa a intensificar o envio de turistas a esta região. O que esta comunidade e empresa turística não esperavam era o acontecimento de uma tragédia natural que destruiu uma parte desta localidade. Diante desta situação, a empresa turística percebeu que devido às circunstâncias deveria reduzir os passeios turísticos a esta localidade durante o restabelecimento da mesma e que esta decisão só iria afetar uma parte desta comunidade, pois a outra parte continuaria servindo de estrutura para os turistas. Em contrapartida, a comunidade considera esta atitude errada e egoísta e que esta decisão poderá causar desemprego para muitas famílias que dependem do turismo e que neste momento precisam destes visitantes para reerguer a parte afetada.

Ação: A empresa turística decidiu reduzir o envio de turistas a esta comunidade enquanto a mesma não seja restabelecida

\section{Cenário 2:}

Um agente de turismo acaba de ser contratado por uma agência de viagens para operar na venda de passagens aéreas. Este operador coincidentemente, no seu primeiro dia de emprego, obteve a oportunidade de fazer negócio a partir da indicação de um amigo, que recomendou a venda de passagens para seus pais e seus tios (04 pessoas) que iriam sair de férias para o Caribe ainda em dez meses. Entretanto, este mesmo agente, a poucos minutos antes de saber da realização deste negócio, havia recebido de seu chefe um comunicado sobre uma promoção de passagens aéreas que teria início no dia seguinte para este mesmo destino. O agente de turismo já presenciou, por parte de outros agentes, finalizações de negócios em situações parecidas e entende que a conclusão deste negócio resultará em uma boa visibilidade por parte de seus superiores. Até porque tal atitude só irá prejudicar financeiramente poucas pessoas.

Ação: O agente de turismo concluiu o negócio.

\section{Cenário 3:}

O proprietário de conhecido resort recebeu incentivos ficais para montar uma filial em uma pequena cidade com reserva de mata atlântica. Os governantes desta localidade perceberam no turismo uma importante fonte de renda que contribuiria para a geração de emprego, lazer e investimentos em infra-estrutura, pois o resort se comprometeu em gerar benefícios para a área em seu entorno. O proprietário do resort ficou interessado em construir tal empreendimento em uma região que ele desconhecia. O único problema para a instalação deste empreendimento está na área em que este será construído, visto que a única extensão de terra propícia para o seu estabelecimento é a área da reserva de mata atlântica. O empresário diante deste contexto propôs construir o resort sem prejudicar a mata. Todavia, os moradores da cidade e organizações de preservação ambiental alegam que mesmo que o empreendimento se comprometa a preservar a mata ela sofrerá algum tipo de dano, mesmo sendo ele pequeno e não imediato, pois a fauna e a flora acabam sentindo ao longo dos anos o efeito da presença dos visitantes, bem como das instalações. Além disso, argumenta-se que a presença do resort, nesta área de reserva, irá prejudicar a ventilação da população próxima a ele.

O empresário do empreendimento, bem como os governantes da cidade estão cientes da necessidade ambiental de preservar tal reserva, porém percebem que os benefícios gerados a esta cidade são superiores a probabilidade da ocorrência de tais efeitos negativos alegados pelos moradores e ambientalistas.

Ação: O empresário resolve construir o resort na área da reserva com o consentimento dos governantes da cidade.

$$
\begin{gathered}
\text { Quadro } 1 \text { - Cenários desenvolvidos } \\
\text { Fonte: Elaboração própria }
\end{gathered}
$$

Os mesmos autores Wason, Polonsky e Hyman (2002) também indicam a necessidade de submeter os cenários a avaliações de especialistas para avaliar a adequação do conteúdo dos cenários ao propósito da escala no intuito de realizar a validação de conteúdo e face. Os 
cenários e itens da escala foram então submetidos a avaliações por seis acadêmicos, que foram convidados a julgar a adequação do cenário em uma escala de 5 pontos (de (1) totalmente inadequado a (5) totalmente adequado) nos seguintes critérios: 1 - evidência das seis características da intensidade moral, 2 - presença de dilema ético, 3 - desenvolvimento do cenário, 4 - clareza na compreensão do cenário, 5 - linguagem do cenário e 6 representação do contexto real.

As médias indicaram que as avaliações de adequação ficaram entre 'bastante adequado' e 'totalmente adequado'. Neste sentido, a conclusão preliminar dos autores deste artigo foi de que os cenários estavam válidos em dois critérios de base de sua validade (conteúdo e face).

\subsection{Itens da escala}

Para cada um dos cenários desenvolvidos o respondente teve de se posicionar em relação, a itens relacionados às seis características da intensidade moral. Entretanto, é importante destacar que para a elaboração dos itens da escala de intensidade moral, foram tomados como referência diversas pesquisas de ética em negócios em marketing que estudaram e operacionalizaram quantitativamente o construto intensidade moral, como as que seguem: Singhapakdi, Vitell e Kraft (1996), Marshall e Dewe (1997), Frey (2000), Paolillo e Vitell (2002), May e Pauli (2002), Kini, Ramakrishana e Vijayaraman (2004), Marta e Singhapakdi (2005), McMahon e Harvey (2006), Vitell e Patwardhan (2008), Sweeney e Costello (2009) e Carlson, Kacmar e Wadsworth (2009).

A investigação mais detida destes estudos e da natureza do construto demonstraram o uso de escalas de apenas um item como as de Singhapakdi, Vitell e Kraft (1996), Frey (2000), Marta e Sighapakdi (2005) e McMahon e Harvey (2006). Este procedimento atende inclusive à prescrições especializadas sobre mensuração que procuram superar o paradigma psicométrico convencional (DROLET; MARRISON, 2001; KWON; TRAIL, 2005; BERGKVIST; ROSSITER, 2007). Como procedimento de verificação, foi adotada a escala do tipo phrase completion de 11 pontos, conforme proposto por Hodge e Gillespie (2007).

Os itens construídos foram enunciados como segue (entre parênteses, pontos de referência da escala phrase completion): Magnitude das consequências - Para mim, o malefício final resultante da ação tomada pela empresa turística será ('Pouco significativo', 'Razoavelmente significativo', 'Muito significativo'); Consenso social - Acredito que existiria um consenso, 
por parte da sociedade, de que a ação tomada pela empresa seria ('Pouco correta', 'Razoavelmente correta', 'Muito correta'); Probabilidade de efeito - Acredito que a probabilidade de se gerar um malefício pela ação da empresa turística seja ('Pouco significativa', 'Razoavelmente significativa', 'Muito significativa'); Imediatismo temporal Para mim, o dano imediato provocado pela ação da empresa seria ('Insignificante', 'Razoavelmente significativo', 'Muito significativo'); Proximidade - Para mim, como a empresa turística apresenta proximidade às vítimas em potencial, a ação é ('Pouco correta'; 'Razoavelmente correta'; 'Muito correta'); Concentração do efeito - Acredito que os prejuízos causados pela ação desta empresa turística irão prejudicar ('Poucas pessoas', 'Um número razoável de pessoas', Muitas pessoas') (com exceção do item de proximidade, os demais itens estão indicados no apêndice 2 na forma como apareceram no questionário).

Além das seis características da intensidade moral a escala apresentou um item que remetia à intenção ética dos entrevistados, com o seguinte enunciado "Se eu fosse à empresa turística agiria de forma..." e a escala de phrase completion tinha como pontos de referência : "totalmente contrária"; "próxima"; "totalmente igual". Em seguida, e com base nos cenários e neste conjunto de itens, foi construído um questionário, que continha, além destas questões, mais um conjunto de itens relacionados à aspectos sócio-demográficos.

Estes itens desenvolvidos também foram submetidos a avaliações por seis acadêmicos, aos quais foi solicitado que indicassem em uma escala de 5 pontos, a adequação à definição e a clareza do enunciado. Os resultados sugeriram validade de conteúdo e face dos itens, o que assegurou condições para o passo seguinte.

\subsection{Primeira amostra - estudantes}

A primeira amostra foi constituída por um grupo de 137 estudantes graduandos do curso de turismo de uma universidade pública do Nordeste brasileiro. A coleta foi realizada diretamente em sala de aula, e, dentre as características gerais dos respondentes, observou-se que a maior parte $(66,4 \%)$ era do sexo feminino, solteira $(95,6 \%)$, com faixa etária até 24 anos (77\%) e renda familiar mensal abaixo de $\mathrm{R} \$ 3.000,00$ (64\%).

Os resultados para os três cenários, com a indicação das médias e desvios padrões podem ser visualizados na tabela 1 . As variações sugerem que os cenários e a maioria dos itens conseguiram apreender as diferenças dos três dilemas, algo que é relevante do ponto de vista 
da construção da escala e do teste das relações. Em geral, é possível verificar que o Cenário 1 foi o que apresentou as médias mais próximas do ponto intermediário da escala (5), enquanto que nos Cenários 2 e 3 as médias oscilaram. Apesar da oscilação das médias, o Cenário 3 foi aquele em que os respondentes apresentaram um posicionamento mais ético na lógica dos itens definidos, talvez devido ao dilema envolver um situação ambiental. Ou seja, o cenário ambiental foi o que representou a circunstância de maior conteúdo antiético, no ponto de vista dos entrevistados.

Tabela 1 - Medidas para os três cenários

\begin{tabular}{l|c|c|c|c|c|c}
\hline \multicolumn{1}{c|}{ Variável } & Média1* & Média2 & Média3 & Desvio1 & Desvio2 & Desvio3 \\
\hline Magnitude das consequências & 6,86 & 5,85 & 7,47 & 2,37 & 2,49 & 2,51 \\
Consenso social & 5,10 & 3,98 & 2,99 & 2,67 & 2,39 & 2,46 \\
Probabilidade de efeito & 6,01 & 5,85 & 7,05 & 2,48 & 2,22 & 2,51 \\
Imediatismo temporal & 6,84 & 5,22 & 6,43 & 2,11 & 2,44 & 2,79 \\
Proximidade & 5,14 & 2,91 & 3,18 & 2,81 & 2,43 & 2,42 \\
Concentração do efeito & 6,64 & 4,12 & 7,36 & 2,04 & 2,59 & 2,58 \\
\hline
\end{tabular}

* Legenda: aqui os números indicaram os cenários 1,2 e 3.

Fonte: Dados da pesquisa

Após estas verificações, procedeu-se à extração da correlação entre os itens, e os resultados estão indicados na tabela 2, a partir da qual é possível perceber que muitas das correlações entre as variáveis da intensidade moral foram não nulas, e há evidentemente um efeito do conteúdo do cenário no relacionamento entre as características. Chamaram a atenção os resultados das variáveis probabilidade de efeito, que apresentou correlações elevadas com magnitude das consequências nos três cenários, e a 'proximidade' que apresentou correlações nula ou negativa com magnitude das consequências. Estas situações são indicativas de que, provavelmente, os itens que mensuram estas duas variáveis não estejam conseguindo captar o seu real sentido, segundo o conceito desenvolvido por Jones (1991). 
Tabela 2 - Correlação para os três cenários turísticos

\begin{tabular}{c|c|c|c|c|c|c}
\hline Dimensão & Cenários & Mag. & Cons. & Prob. & Imed. & Proxi. \\
\hline \multirow{3}{*}{ Cons. } & Cenário 1 & $0,044^{*}$ & & & & \\
& Cenário 2 & $-0,284$ & & & & \\
& Cenário 3 & $-0,046^{*}$ & & & & \\
\hline \multirow{3}{*}{ Prob. } & Cenário 1 & 0,567 & $-0,145^{*}$ & & & \\
& Cenário 2 & 0,703 & $-0,234$ & & & \\
& Cenário 3 & 0,799 & $0,023^{*}$ & & & \\
\hline \multirow{3}{*}{ Imed. } & Cenário 1 & 0,506 & $-0,110^{*}$ & 0,464 & & \\
& Cenário 2 & 0,643 & $-0,222$ & 0,657 & & \\
& Cenário 3 & 0,508 & $-0,164^{*}$ & 0,454 & & \\
\hline \multirow{2}{*}{ Proxi. } & Cenário 1 & $-0,098^{*}$ & 0,593 & $-0,238$ & $-0,074^{*}$ & \\
& Cenário 2 & $-0,306$ & 0,509 & $-0,311$ & $-0,318$ & \\
& Cenário 3 & $-0,460$ & 0,328 & $-0,382$ & $-0,372$ & \\
\hline \multirow{2}{*}{ Conc. } & Cenário 1 & 0,243 & $-0,222$ & 0,434 & 0,356 & $-0,203$ \\
& Cenário 2 & 0,474 & $-0,083 *$ & 0,507 & 0,496 & $-0,201$ \\
& Cenário 3 & 0,554 & $-0,109 *$ & 0,527 & 0,561 & $-0,418$ \\
\hline
\end{tabular}

Legenda: Mag - Magnitude das consequências, Cons - Consenso social, Prob - Probabilidade de efeito, Imed Imediatismo temporacl, Proxi - proximidade, Conc - Concentração do efeito.

Fonte: Dados da pesquisa

Ao se analisar os resultados desta primeira amostra, conclui-se que, os cenários, não foram fatores de dificuldade na geração de respostas dos respondentes. Adicionalmente, as variações de opiniões, no que se refere aos contextos apresentados, sugerem que os mesmos possuem diferentes perspectivas de conteúdo de intensidade moral, o que é positivo na medida em que os cenários possibilitam a captação de variações de posicionamento em uma amostra heterogênea, o que é indicativo de sua adequação para aferição dos tópicos sob análise Assim, há evidências que sinalizam a coerência e o bom ajustamento dos cenários ao ponderar as sugestões propostas por Wason, Polonsky e Hyman (2002). Desta forma, a necessidade de alterá-los e aperfeiçoá-los não foi requerida.

Considerando os itens da escala, a análise do conteúdo não indicou problemas com a variável probabilidade e as correlações podem ser reais. Por outro lado, ficou evidente a necessidade de reformular o item sobre a 'proximidade', uma vez que, ao se observar a formulação inicial do item foi possível perceber que este estava gerando um questionamento sobre a ação ser correta ou incorreta e não o fato das pessoas afetadas pela ação serem próximas ou não ao agente. Decidiu-se então alterar o enunciado para "Para mim, o efeito danoso (se houver) proveniente da ação (da empresa turística, do agente de viagem ou do empresário do resort) irá afetar pessoas que são", e os pontos de referência da escala de phrase completion foi de 
"pouco próximas a ela, razoavelmente próximas a ela e muito próximas a ela".

Com exceção ao item "proximidade", as outras variáveis da intensidade moral em análise não indicaram a necessidade de ajustes. Neste sentido, é possível concluir que, em geral, a escala apresentou indícios preliminares de consistência em seus itens. Por outro lado, entendeu-se ser necessário desenvolver uma segunda amostragem, para testar o ajuste no item de proximidade, e para captar avaliações de um contexto mais próximo das decisões efetivas do universo do turismo. Isto foi feito na segunda amostragem, cujos resultados estão indicados a seguir.

\subsection{Segunda amostra - estudantes}

Com a reformulação no questionário do item "proximidade", este foi reaplicado com um novo grupo composto por 103 estudantes graduandos do curso de turismo de uma universidade pública de outro estado do Nordeste brasileiro, e por 61 gestores de turismo de três capitais de estados da mesma região do Brasil (totalizando 164 respondentes).

Para o primeiro grupo adotou-se a mesma estratégia de coleta da primeira amostra (direta em sala de aula), e para os gestores os dados foram coletados de duas formas, sendo estas presencial (52 respondentes) e on-line (9 entrevistados). Com relação às outras características desta segunda amostra de estudantes, verificou-se que estes eram em sua maior parte do sexo feminino $(67,0 \%)$, solteiros $(98,1 \%)$, com faixa etária predominante até 24 anos $(82 \%)$ e renda familiar inferior de $\mathrm{R} \$ 3.000,00(68,0 \%)$. Nos gestores, em relação à característica do nível de escolaridade, $43 \%$ revelaram que apresentavam nível superior completo ou incompleto, sendo a maior parte do sexo masculino $(54,1 \%)$, casados $(54,1 \%)$, com faixa etária acima de 40 anos $(49,2 \%)$.

Os resultados em relação as seis características da intensidade moral para estas duas amostras: estudantes e gestores (Tabela 3), em geral, ficaram próximos do que foi observado na primeira amostragem com a variação esperada na dimensão proximidade e entre estudantes e gestores. Em geral, entende-se que a escala está captando as variações previstas no universo, o que sugere uma consistência da escala, tanto para os cenários quanto para os itens. 
Tabela 3 - Medidas para os três cenários considerando os estudantes e gestores

\begin{tabular}{|c|c|c|c|c|c|c|c|c|c|c|c|c|}
\hline \multirow{2}{*}{ Variável } & \multicolumn{2}{|c|}{ Média1 } & \multicolumn{2}{|c|}{ Média2 } & \multicolumn{2}{|c|}{ Média3 } & \multicolumn{2}{|c|}{ Desvio1 } & \multicolumn{2}{|c|}{ Desvio2 } & \multicolumn{2}{|c|}{ Desvio3 } \\
\hline & Est & Ges & Est & Ges & Est & Ges & Est & Ges & Est & Est. & Est & Ges \\
\hline $\begin{array}{l}\text { Magnitude das } \\
\text { consequências }\end{array}$ & 6,9 & 6,7 & 5,6 & 7,4 & 7,5 & 6,5 & 2,0 & 2,1 & 2,1 & 2,4 & 2,1 & 2,8 \\
\hline Consenso social & 5,4 & 5,4 & 4,0 & 3,4 & 3,1 & 4,4 & 2,5 & 2,7 & 2,6 & 2,8 & 2,3 & 2,7 \\
\hline Probabilidade de efeito & 6,1 & 5,7 & 5,6 & 6,7 & 7,2 & 6,4 & 2,3 & 2,2 & 2,2 & 2,8 & 2,1 & 2,9 \\
\hline Imediatismo temporal & 6,4 & 6,4 & 5,3 & 6,6 & 6,3 & 5,7 & 2,2 & 2,0 & 2,5 & 2,8 & 2,4 & 3,0 \\
\hline Proximidade & 6,2 & 6,1 & 5,3 & 6,3 & 3,5 & 4,9 & 2,7 & 2,3 & 2,9 & 2,4 & 3,3 & 3,3 \\
\hline Concentração do efeito & 6,6 & 6,2 & 3,7 & 4,6 & 7,4 & 6,5 & 2,0 & 2,2 & 2,3 & 2,9 & 2,2 & 3,0 \\
\hline
\end{tabular}

Fonte: Dados da pesquisa

Ao se observar as correlações entre os itens, os resultados, que estão indicados na tabela 4, sugerem também variações em relação aos cenários, havendo alguns casos de correlações nulas e diversas correlações negativas. Comparando estes resultados com aqueles obtidos na primeira amostragem, constata-se que há regularidade em termos de existência de variação dos relacionamentos medidos pela correlação. Adicionalmente, e considerando ainda a primeira amostragem, que foi somente de estudantes, é possível observar que houve variações específicas nas respostas de pares de itens, com casos de correlações que lá foram nulas e aqui não o foram (houve casos de inversão da valência da correlação, mas provavelmente isto ocorreu devido a mudança no enunciado da dimensão proximidade). Isto sugere que há também um efeito associado à natureza do respondente, além da natureza do dilema ético.

Tabela 4 - Correlação para os três cenários de forma agregada

\begin{tabular}{c|c|c|c|c|c|c}
\hline Dimensão & Cenários & Mag. & Cons. & Prob. & Imed. & Proxi. \\
\hline \multirow{3}{*}{ Cons. } & Cenário 1 & $-0,088^{*}$ & & & & \\
& Cenário 2 & $-0,474$ & & & & \\
& Cenário 3 & $-0,406$ & & & & \\
\hline \multirow{3}{*}{ Prob. } & Cenário 1 & 0,606 & $-0,170$ & & & \\
& Cenário 2 & 0,625 & $-0,399$ & & & \\
& Cenário 3 & 0,759 & $-0,379$ & & & \\
\hline \multirow{3}{*}{ Imed. } & Cenário 1 & 0,488 & $-0,120^{*}$ & 0,580 & & \\
& Cenário 2 & 0,712 & $-0,409$ & 0,719 & & \\
& Cenário 3 & 0,659 & $-0,278$ & 0,641 & & \\
\hline \multirow{3}{*}{ Proxi. } & Cenário 1 & $0,143^{*}$ & $0,060^{*}$ & 0,239 & 0,220 & \\
& Cenário 2 & 0,222 & $-0,092^{*}$ & 0,232 & 0,286 & \\
\hline \multirow{2}{*}{ Conc. } & Cenário 3 & $0,051^{*}$ & 0,209 & $-0,032^{*}$ & $0,013^{*}$ & \\
\hline & Cenário 1 & 0,508 & $-0,173$ & 0,500 & 0,484 & 0,222 \\
& Cenário 2 & 0,483 & $-0,269$ & 0,514 & 0,565 & 0,347 \\
& Cenário 3 & 0,648 & $-0,363$ & 0,692 & 0,576 & $-0,040^{*}$ \\
\hline
\end{tabular}

Legenda: Mag - Magnitude das consequências, Cons - Consenso social, Prob - Probabilidade de efeito, Imed Imediatismo temporal, Proxi - Proximidade, Conc - Concentração do efeito.

Fonte: Dados da pesquisa 
A variação por cenário sugere ainda que a correlação entre os itens varia de acordo com a situação ética envolvida, o que parece indicar que os itens não podem ser tidos, a priori, como variáveis refletivas de um mesmo construto latente.

Para retomar os casos que demandaram cuidados na amostra anterior, verificou-se que, na dimensão de probabilidade, mantiveram-se correlações elevadas com a dimensão de intensidade moral, repetindo o padrão antes verificado o que sugere que estas dimensões efetivamente possuem correlação mais alta. Em relação à variável proximidade, ao que parece a alteração no enunciado gerou medidas que parecem estar mais alinhadas com o senso conceitual dos itens.

Desta forma, considerando medidas também da primeira amostragem, os resultados apontam que, embora ocorram correlações elevadas, em nenhum caso os valores foram elevados o suficiente para indicar colinearidade (todas as correlações foram menores que 0,9), o que parece ser um indicador consistente de que não há sobreposição de variáveis e conteúdo. Embora isto não possa ser sinalização de validade discriminante, ao menos no sentido convencional, é possível retirar a validade da escala na medida em que não se verificou que qualquer das variáveis tem a possibilidade de substituir ou explicar as demais em proporção maior que 58\% (que ocorre somente no terceiro cenário entre as variáveis probabilidade e magnitude de consequências).

\subsection{Análise de regressão}

Objetivando a validação adicional da escala foi desenvolvida a análise de regressão tomando por variável dependente a dimensão intenções éticas e variáveis independentes as seis características da intensidade moral, para ambas as amostras (estudantes e gestores) do estudo de maneira agregada. Este procedimento baseia-se no fato de estas influências serem recorrentes na literatura especializada, e que está demonstrado que as medidas de intensidade moral são consistentes previsoras das intenções éticas dos respondentes das pesquisas realizadas. Por esta razão, as intenções éticas podem ser tidas como critério de validação da escala desenvolvida.

Cabe, por outro lado, realçar que nem todas as variáveis de intensidade moral são preditoras de intenções éticas em todas as circunstâncias. Pelo contrário, há dilemas em que determinadas dimensões de intensidade moral exercem ou não influência, e isto vem sendo sistematicamente mostrado na literatura (SINGHAPAKDI; VITELL; KRAFT, 1996; 
MARTA; SIGHAPAKDI, 2005). Deste modo, tomaremos como primeira evidência de validade de critério o fato de o conjunto de dados prever sistematicamente as intenções éticas independente do cenário, e em seguida analisaremos rapidamente as variações dos resultados.

Considerando a análise de regressão, a análise de variância indicou que, em cada cenário, e para os dois grupos, havia sempre ao menos uma variável de influência sobre a dimensão intenções éticas (dados das aferições gerais: cenário $1-\mathrm{R}^{2}=32,7 \%, \mathrm{~F}=12,693, \mathrm{p}<0,001$; cenário $2-\mathrm{R}^{2}=49 \%, \mathrm{~F}=25,162$, $\mathrm{p}<0,001$; cenário $\left.3-\mathrm{R}^{2}=57,6 \%, \mathrm{~F}=35,491, \mathrm{p}<0,001\right)$. Estes resultados deixam evidente que, em conjunto, as variáveis de intensidade moral são preditoras consistentes das intenções éticas, o que converge com a expectativa estabelecida e indica a validade da escala utilizada.

Como exposto na tabela 5, as indicações de influência variaram de acordo com o cenário e com o grupo de referência da pesquisa. Somente a dimensão de consenso social se mostrou como preditora das intenções éticas independente do cenário, ao passo que a probabilidade de efeito foi preditora em dois dos cenários, e imediatismo temporal, concentração do efeito e magnitude das consequências foram previsores em um cenário cada.

Tabela 5 - Correlação para os três cenários de forma agregada

\begin{tabular}{l|c|c|c|c|c|c|c|c|c}
\hline \multirow{2}{*}{ Variáveis } & \multicolumn{3}{c|}{ Cenário 1 } & \multicolumn{3}{c|}{ Cenário 2 } & \multicolumn{3}{c}{ Cenário 3 } \\
\cline { 2 - 9 } \multicolumn{1}{c|}{ Beta } & $\mathbf{T}$ & Sig. & Beta & $\mathbf{t}$ & Sig. & Beta & t & Sig. \\
\hline Magnitude das consequências &,- 098 & $-1,117$ &, 266 &,- 142 & $-1,633$ &, 104 &,- 215 & $-2,414$ & $\mathbf{, 0 1 7}$ \\
Consenso social &, 471 & 6,983 & $\mathbf{, 0 0 0}$ &, 555 & 8,461 & $\mathbf{, 0 0 0}$ &, 293 & 4,934 & $\mathbf{, 0 0 0}$ \\
Probabilidade de efeito &, 038 &, 406 &, 685 &,- 182 & $-2,121$ & $\mathbf{, 0 3 5}$ &,- 254 & $-2,835$ & $\mathbf{, 0 0 5}$ \\
Imediatismo temporal &,- 224 & $-2,647$ & $\mathbf{, 0 0 9}$ &,- 032 &,- 332 &, 740 &,- 050 &,- 685 &, 494 \\
Proximidade &, 020 &, 289 &, 773 &, 013 &, 212 &, 832 &, 070 & 1,295 &, 197 \\
Concentração do efeito &,- 022 &,- 271 &, 787 &, 185 & 2,553 & $\mathbf{, 0 1 2}$ &,- 129 & $-1,695$ &, 092 \\
\hline
\end{tabular}

Fonte: Dados da pesquisa

\subsection{Discussão dos resultados}

Como resultado da primeira amostragem verificou-se que os entrevistados entenderam o cenário 3 como sendo o de maior conteúdo antiético, ficando o dilema social em um ponto intermediário. Em relação a análise de correlação, foi possível observar a necessidade de alteração do questionamento do item referente a variável proximidade.

Considerando a segunda amostragem, composta de estudantes e gestores da atividade turística, as médias indicaram que para o cenário de contexto econômico o mesmo se apresentou como sendo mais antiético para os gestores do que para os estudantes. Já em 
relação ao dilema ambiental, os resultados das médias sugeriram que os gestores percebem menos problemas éticos em comparação com a amostra de estudantes. Também como resultados desta amostragem averiguou-se oscilações nas percepções dos entrevistados considerando a natureza dos cenários, bem como correlações mais ajustadas após a adequação do item da variável "proximidade"

Em geral, para as duas amostragens é possível verificar que tanto os cenários quanto os itens mostraram-se consistentes enquanto proposta de mensuração do construto intensidade moral no contexto da decisão gerencial do gestor de turismo. As etapas preliminares de que haviam sugerido as validades de conteúdo e de face dos cenários e dos itens tiveram seus resultados quase que totalmente reafirmados nas amostragens realizadas, com uma alteração apenas efetuada no item de 'proximidade'.

Como estratégia adicional de validação, utilizamos a variável intenções éticas para verificar a validade de critério, tendo observado efetivamente que o conjunto de variáveis mantém a relação de previsão conforme esperado, embora os itens de previsão variem de acordo com o dilema ético associado, que é algo esperado tendo em vista a literatura pesquisada. Isto reforça os indícios de validade da escala, e se soma aos resultados de conteúdo e face indicados. Neste sentido, em nosso entendimento, os itens que compõem a intensidade moral são dimensões conceitualmente independentes e distintas, que podem variar conjuntamente a depender do dilema ético envolvido.

\section{Considerações Finais}

Neste artigo foi desenvolvida uma proposta de escala para mensuração do construto intensidade moral especificamente para o setor do turismo. Embora a intensidade moral seja um tema amplamente estudado na literatura internacional, entende-se que poucos estudos podem ser aplicados de forma apropriada à atividade turística, e isto justifica o esforço desenvolvido neste estudo. Além do aspecto associado ao contexto empírico também foi feito o esforço de trazer para a análise alguns dos procedimentos metodológicos e operacionalizações estatística da teoria da mensuração.

Em geral, os testes empíricos indicaram a consistência dos itens e dos cenários desenvolvidos. Desta forma, a proposta ficou consolidada em três cenários com características comumente 
vivenciadas por gestores da atividade turística, e ainda em seis itens, cada um direcionado a medir uma característica definida da intensidade moral.

Este instrumento pode ser aplicado tanto para averiguações exploratórias e comparativos de contextos distintos da atividade turística (segmentos ou espaços geográficos), e pode servir ainda para a aplicação com outros construtos para efeito de teste de hipóteses de relacionamentos da intensidade moral com outros construtos.

Algumas limitações são evidenciadas na pesquisa, a começar pela natureza não probabilística das amostragens, e pela limitação do tamanho das amostras. Segue disto a recomendação da realização de outras amostragens (inclusive em diferentes contextos geográficos) e procedimentos preferencialmente probabilísticos. Adicionalmente, as alternativas de validação adotadas podem ser aperfeiçoadas, apesar de coerentes com as novas indicações da teoria da mensuração. Nestes termos, o teste da escala e das relações entre construtos em rede nomológica seria um tema de pesquisa interessante e de consolidação da proposta de escala.

A expectativa é de que este estudo tenha real aplicação no contexto de prática gerencial e da formação de futuros profissionais da área do turismo. Nestes termos, é recomendado que, no nível de formação, as escolas de turismo utilizem o instrumento para averiguar o nível de sensibilidade ética de seus estudantes como forma de promover uma formação profissional melhor alinhada a princípios éticos sociais e profissionalmente aperfeiçoado. No nível de desenvolvimento profissional, recomenda-se que as instituições que congregam gestores e profissionais de turismo e mesmo organizações do setor utilizem o instrumento como referencial de identificação de sensibilidade e de tendências éticas, o que pode representar informações para desenvolvimento de ações de formação e aperfeiçoamento profissional.

\section{Referências}

BERGKVIST, L.; ROSSITER, J. R. The predictive validity of multiple-item versus single-item measures of the same constructs. American Marketing Association, v. 44. p. 175-184, 2007.

CARLSON, D. S.; KACMAR, K. M.; WADSWORTH, L. L. The impact of moral intensity dimensions on ethical decision-making: Assessing the relevance of orientation. Journal of Managerial Issues, v. 21, n. 4, p. 534-551, 2009.

CHERRY, John; FRAEDRICH, John. Perceived risk, moral philosophy and marketing ethics: Mediating influences on sales managers' ethical decision-making. Journal of Business Research, n.55, pp. $951-962,2002$. 
CHURCHILL JR, G. A. Paradigm for developing better measures of marketing constructs. Journal of Marketing Research, v. 16, p. 64-73, 1979.

DIAMANTOPOULOS, A.; WINKLHOFER, H. M. Index construction with formative indicators: an alternative to scale development. Journal of Marketing Research, v. 38, pp. 269-277, 2001.

DROLET, A. L; MARRISON, D. G. Do we really need multiple-item measures in service research? Journal of Service Research, v. 3, pp. 196-204, 2001.

FERRELL, O. C; GRESHAM, L. G. A contingency framework for understanding ethical decision making in marketing. Journal of marketing, v. 49, pp. 87-96, 1985.

FREY, B. F. The impact of moral intensity on decision making in business context. Journal of Business Ethics, n. 26, pp. 181-195, 2000.

HERINGTON, Carmel; WEAVEN, Scott. Does marketing attract less ethical students? An assessment of the moral reasoning ability of undergraduate marketing students. Journal of Marketing Education, n. 29 , pp. $154-163,2007$.

HIGGINS-DESBIOLLES, F. More than an "industry": the forgotten power of tourism as a social force. Tourism Management, n. 27, pp. 1192-1208, 2006.

HODGE, D. R; GILLESPIE, D. F. Phrase completion scales: A better measurement approach than Likert scales? Journal of Social Service Research, v. 33, n. 4, p. 1-12, 2007.

HOJEVAC, I. A. Business ethics in tourism - as a dimension of TQM. Total Quality management, v. 19, n. 10, pp. 1029-1041, 2008.

HOLDEN, A. In need of new environmental ethics for tourism? Annals of Tourism Research, v. 30, n.1, pp. 94-108, 2003.

HUNT, S. D; VITELL, S. J. A general theory of marketing ethics. Journal of Macromarketing, v. 6, n. 5, p. 5-16, 1986.

JONES, T. M. Ethical decision making by individuals in organizations: An issue-contingent model. Academy of Managementt Review, v. 16, n. 2, p. 366-395, 1991.

KINI, R. B; RAMAKRISHNA, H. V; VIJAYARAMAN, B. S. Shaping of moral intensity regarding software piracy: A comparison between Thailand and U. S. students. Journal of Business Ethics, n. 43. pp. 91-104, 2004.

KWON, H; TRAIL, G. The feasibility of single-item measures in sport loyalty research. Sport Management Review, n. 8, pp. 69-89, 2005.

LANSING, P.; VRIES, P. De. Sustainable tourism: Ethical alternative or marketing ploy? Journal of Business Ethics, n. 72, pp. 77-85, 2007.

MARSHALL, D.; DEWE, P. An investigation of the components of moral intensity. Journal of Business Ethics, v. 16, pp. 521-529, 1997.

MARTA, J. K. M; SINGHAPAKDI, A. Comparing Thai and US businesspeople. Perceived intensity of unethical marketing practices, corporate ethical values, and perceived importance of ethics. International Marketing Review, v. 22, n. 5, pp. 562-577, 2005.

MAY, D. R; PAULI, K. P. The role of moral intensity in ethical decision making: A review and investigation of moral recognition, evaluation, and intention. Business and society, n. 41, pp. $84-117$, 2002.

MCMAHON, J. M; HARVEY, R. J. An analysis of the factor structure of Jones's moral intensity construct. Journal of Business Ethics, n. 64, pp. 381-404, 2006.

OPPERMANN, M. Sex tourism. Annals of Tourism Research, v. 26, n. 2, p. 251-266, april, 1999. 
PAOLILLO, J. G. P; VITELL, S. J. An empirical investigation of the influence of selected personal, organizational and moral intensity factors on ethical decision making. Journal of Business Ethics, $\mathrm{n}$. 35, pp. 65-74, 2002.

ROSSITER, J. R. The C-OAR-SE procedure for scale development in marketing. International Journal of Research in Marketing, v. 19, n. 4, pp. 305-335, 2002.

SINGHAPAKDI, A.; VITELL, S. J; KRAFT, K. L. Moral intensity and ethical decision-making of marketing professionals. Journal of Business Research, n. 36, pp. 245-255, 1996.

SIRAKAYA, Ercan; WOODSIDE, Arch. G. Building and testing theories of decision making by travellers. Tourism Management, n. 26, pp. 815 - 832, 2005.

SMALlMAN, Clive; MOORE, Kevin. Process studies of tourists' decision-making. Annals of Tourism Research, v. 37, n. 2, pp. 397 - 422, 2010.

SWEENEY, B.; COSTELLO, F. Moral intensity and ethical decision-making: an empirical examination of undergraduate accounting and business students. Accounting Education: An International Journal, v. 18, n.1, pp. 75-97, 2009.

TREVIÑO, L. K. Ethical decision making in organizations: a person-situation interactionist model. Academy of Management Review, v. 11, n. 3, p. 601-617, 1986.

VITELL, S. J; PATWARDHAN, A. The role of moral intensity and moral philosophy in ethical decision making: a cross-cultural comparison of China and the European Union. Business Ethics: A European Review, v. 17, n. 2, pp. 196-209, 2008.

VITELL, Scott. J; SINGHAPAKDI, Anusorn; THOMAS, James. Consumer ethics: An application and empirical testing of the Hunt-Vitell theory of ethics. Journal of Consumer Marketing, v. 18, n. 2, pp. $153-178,2001$.

WASON, K. D; POLONSKY, M. J; HYMAN, M. R. Designing vignette studies in marketing. Australasian Marketing Journal, v. 10, n. 3, pp. 41-87, 2002.

WEBB, Deborah. J; MOHR, Lois. A; HARRIS, Katherine. E. A re-examination of socially responsible consumption and its measurement. Journal of Business Research, n. 61, pp. 91 - 98, 2008

WEEDEN, C. Ethical tourism: an opportunity for competitive advantage? Journal of Vacation Marketing, v. 8, n.2, pp. 141-153, 2002.

\section{Recebido em: 27/12/2011}

Aprovado em: 28/05/2012 


\section{Apêndice 1 - Variáveis no Cenário}

\begin{tabular}{|c|c|}
\hline \multicolumn{2}{|r|}{ CENÁRIO 1} \\
\hline Característica & Evidência no cenário \\
\hline $\begin{array}{l}\text { Magnitude das } \\
\text { consequências }\end{array}$ & $\begin{array}{l}\text { O resultado final da ação recairá sobre as pessoas que dependem do } \\
\text { turismo }\end{array}$ \\
\hline Consenso social & A comunidade considera a ação da empresa errada e egoísta \\
\hline Probabilidade de efeito & A ação da empresa provavelmente causará desemprego \\
\hline Imediatismo temporal & $\begin{array}{l}\text { A ação da empresa durará o tempo necessário para que a comunidade } \\
\text { se restabeleça }\end{array}$ \\
\hline Proximidade & Há um sentimento amigável entre a empresa e a comunidade \\
\hline Concentração do efeito & A ação irá afetar parte da comunidade \\
\hline \multicolumn{2}{|r|}{ CENÁRIO 2} \\
\hline Característica & Evidência no cenário \\
\hline $\begin{array}{l}\text { Magnitude das } \\
\text { consequências }\end{array}$ & $\begin{array}{l}\text { O saldo final dos malefícios e/ou benefícios resultantes da ação } \\
\text { recairá sobre poucas pessoas }\end{array}$ \\
\hline Consenso social & $\begin{array}{l}\text { A atitude do agente parece ser comum entre outros profissionais } \\
\text { desta área }\end{array}$ \\
\hline Probabilidade de efeito & A ação irá causar dano financeiro para os viajantes \\
\hline Imediatismo temporal & As pessoas irão viajar ainda em 10 meses \\
\hline Proximidade & As pessoas que irão viajar são os pais e tios de um amigo do agente \\
\hline Concentração do efeito & Efeito sobre 4 pessoas \\
\hline \multicolumn{2}{|r|}{ CENÁRIO 3} \\
\hline Característica & Evidência no cenário \\
\hline $\begin{array}{l}\text { Magnitude das } \\
\text { consequências }\end{array}$ & Algum dano pode ocorrer à reserva mesmo que seja pequeno \\
\hline Consenso social & $\begin{array}{l}\text { Os moradores da cidade e as organizações de preservação ambiental } \\
\text { estão descontentes com a construção do resort }\end{array}$ \\
\hline Probabilidade de efeito & Há a probabilidade da geração de alguns danos \\
\hline Imediatismo temporal & O dano da ação poderá ser sentido com o decorrer dos anos \\
\hline Proximidade & O proprietário do resort não conhecia a região \\
\hline Concentração do efeito & $\begin{array}{l}\text { A construção do resort irá prejudicar a fauna e flora da reserva e a } \\
\text { população próxima a ele com a redução da ventilação }\end{array}$ \\
\hline
\end{tabular}




\section{Apêndice 2 - Itens da Escala de Intensidade Moral Final}

1 - Magnitude das consequências - Para mim, o malefício final resultante da ação tomada pela empresa turística será

Pouco significativo

Razoavelmente significativo

Muito significativo

\begin{tabular}{l|l|l|l|l|l|l|l|l|l|l|l|l}
0 & 1 & 2 & 3 & 4 & 5 & 6 & 7 & 8 & 9 & 10 \\
\hline
\end{tabular}

2 - Consenso social - Acredito que existiria um consenso, por parte da sociedade, de que a ação tomada pela empresa seria

Pouco correta
\begin{tabular}{c|c|c|c|c|c|c|c|c|c|c|}
0 & \multicolumn{1}{c}{ Razoavelmente correta } \\
0
\end{tabular}

3 - Probabilidade de efeito - Acredito que a probabilidade de se gerar um malefício pela ação da empresa turística seja

\begin{tabular}{c|c|c|c|c|c|c|c|c|c|c|c|} 
Pouco significativa & \multicolumn{1}{c}{$\begin{array}{c}\text { Razoavelmente significativa } \\
0\end{array}$} & 1 & 2 & 3 & 4 & 5 & 6 & 7 & 8 & 9 & 10 \\
\hline
\end{tabular}

4 - Imediatismo temporal - Para mim, o dano imediato provocado pela ação da empresa seria Insiginificante

Razoavelmente significativo

Muito significativo

$4+5$

\begin{tabular}{l|l|l}
7 & 8 & 9
\end{tabular}

10

5 - Proximidade - Para mim, o efeito danoso (se houver) proveniente da ação (da empresa turística, do agente de viagem ou do empresário do resort) irá afetar pessoas que são

Pouco próximas a ela

Razoavelmente próximas a ela

Muito próximas a ela

\begin{tabular}{l|l|l|l|l|l|l|l|l|l|l|l|l|l}
0 & 1 & 2 & 3 & 4 & 5 & 6 & 7 & 8 & 9 & 10 \\
\hline
\end{tabular}

6 - Concentração do efeito - Acredito que os prejuízos causados pela ação desta empresa turística irão prejudicar

Poucas pessoas

Um número razoável de

Muitas pessoas

\begin{tabular}{|l|l|l|l|l|l|l|l|l|l|l|l}
0 & 1 & 2 & 3 & 4 & 5 & 6 & 7 & 8 & 9 & 10 \\
\hline
\end{tabular}

